\title{
Discontinuous User-Energy Distribution for Dynamically Ordered Successive Interference Cancellation
}

\author{
Francesc Molina, Student Member, IEEE and Josep Sala-Álvarez, Senior Member, IEEE
}

\begin{abstract}
In this letter, we consider many users sharing the same codebook and employing random signatures to modulate preamble plus payload data. Successive interference cancellation (SIC) is performed at the receiver side, whereby all user-strengths are estimated by preamble cross-correlations to set the userdecoding order, after which SIC operates. Our contribution in the infinite-user regime is twofold. Firstly, the innovative application of variational calculus to derive the asymptotic user-energy distribution that maximizes SIC's performance. Contrarily to exponentially decaying distributions obtained under perfectly ordered SIC, we derive stationary points with piecewise constant structures as potential locally-optimal solutions for the spectral efficiency maximization of estimation-based SIC. Secondly, the proposal of a suboptimal algorithm to compute locally-optimal solutions with high performance/complexity ratio.
\end{abstract}

Index Terms-Massive multiple access, dynamically ordered successive interference cancellation, variational calculus.

\section{INTRODUCTION}

$\mathbf{T}$ HE multiple access (MA) channel consists of many users transmitting simultaneously towards the same destination. In orthogonal MA, users are decoded independently in time, frequency or code domains. Nevertheless, in crowded settings, the simultaneous operation of all users is unfeasible due to the scarcity of orthogonal resources. In non-orthogonal MA (NOMA), users exploit the same collision domain. The system supports a higher number of users when the excess of interference is resolved through interference cancellation [1].

In this context, a number of multiuser detection techniques can be adopted to provide reliable MA [2], [3]. Such is the case if the central node exploits successive interference cancellation (SIC). In this case, when users, instead of using several rates [4], employ the same codebook, as in unsourced NOMA [5], the optimal user-decoding order (UO) entails the SIC operation from the strongest to the weakest user [6]. However, in large NOMA settings, the receiver may not know the strength of each user. Thus, the optimal UO is substituted by a UO based on statistical CSI [7] when partial knowledge is available, or on strengths estimation in the absence of such knowledge [8].

This letter concentrates on the latter case, whose successive decoding operation is referred to as dynamically (estimationbased) ordered SIC. This applies, for instance, in unsourced NOMA settings [5] where user identities are unknown, or in random access satellite networks handling such number of users that perfect knowledge of received powers is not

Work funded in part by Agencia Estatal de Investigación and by European Regional Development Fund under projects TEC2016-76409-C2-1-R (WIN TER) and PID2019-105717RB-C22 (RODIN), and in part by the Catalan administration (AGAUR) under Grant 2017 SGR 578. F. Molina is also supported by predoctoral Fellowship 2020FI_B2 00062 (AGAUR).

The authors are with the Department of Signal Theory and Communications, Universitat Politècnica de Catalunya, 08034 Barcelona, Spain (e-mail francesc.molina@upc.edu; josep.sala@upc.edu). feasible [9]. Some authors have studied, for few users, this SIC's outage performance as in [8]. Nowadays, the handicap is to cope with large systems where difficulty resides in distinguishing the strongest user among very many of them.

In the satellite context, several energy allocations have been investigated to improve the spectral efficiency (SE) of massive MA networks under random spreading waveforms from both theoretical [10], [11] and practical sides [12], [13]. From a theoretic view, the SE-optimum user-energy distribution under perfectly ordered SIC is shown exponential for ideal encodingcancellation schemes in [10], and practically exponential for non-ideal schemes in [11]. Several allocations under more practical SIC systems are designed for imperfect SIC in [12], [13]. In all cases, however, the authors take up the optimal UO that coincides with the order of user symbol energies.

In this work, we adopt a SIC receiver for which symbol energies from all users are unknown. We analyze a network handling many users in the asymptotic large-user regime, where users employ the same encoder-decoder, whose known error characteristic shows relevant discrepancies relative to that in the packet-asymptotic case, followed by random signature waveforms. We answer how the most favorable theoretic userenergy distribution changes when prior to SIC, symbol energies of all users are estimated by preamble cross-correlations and are used to set the UO. We leverage the system model derived in [14] to analyze the former SIC. Our main result proves, via variational calculus, that contrarily to exponential user-energy distributions obtained in [10], [11], piecewise constant functions constitute the stationary points of the SE maximization of dynamically ordered SIC. Competitive local optimums are found using a low complexity algorithm.

Section II states the system model. Section III derives largeuser results. Section IV presents the asymptotic optimization with results in Section V. Section VI offers conclusions.

Notation: The over-dot $\dot{x}$ is used for differentiation. Partial derivatives of $G\left(x_{1}, \ldots, x_{n}\right)$ are denoted either $\partial_{x_{i}} G$ or $G_{x_{i}}$.

\section{SySTEM MODEL}

We evaluate a large NOMA setting where $K$ users transmit $n_{p}$-symbol packets superposed in the code domain. The packet structure is divided into $n_{o}$ preamble plus $n_{e}$ payload symbols. For payload generation, users use the same codebook [13] and transmit at $R$ bits per channel use at symbol period $T_{s}$. The symbol energy for user $k$ is $E_{\mathrm{x}}[1 \leq k \leq K]$. User $k$ signs symbol $m, s_{k}[m]$, using the unit-energy waveform $c_{k, m}(\mathrm{t})$ made up of a long pseudorandom code (with period outweighing many symbols). The waveforms $c_{k, m}(\mathrm{t})$ are non-orthogonal to each other with second moment of the cross-correlation $\frac{1}{N}$.

Communication takes place over slowly time-varying flatfading channels, e.g. in a satellite return link with long 
coherence times and limited peak-to-average power ratio per user. The channel gain for user $k$ is $h[k]$, and the received symbol energy from the same user $E_{\mathrm{r}}[k]=E_{\mathrm{x}}[k] h[k]$. The received signal aggregates the transmitted packets $a_{k}$ under the (delayed) symbol signatures, and is corrupted by additive Gaussian noise $w(\mathrm{t})$ as:

$$
y(\mathrm{t})=\sum_{k=1}^{K} \sqrt{E_{\mathrm{r}}[k]} \sum_{m=0}^{n_{p}-1} a_{k}[m] c_{k, m}\left(\mathrm{t}-m T_{s}-\tau[k]\right)+w(\mathrm{t})
$$

Since users employ non-orthogonal spreading waveforms, interference is superimposed after the symbol matched filter of each user. We leverage SIC in conjunction with energy allocation to provide reliable MA. In this massively populated setting, we adopt, unlike [10], [11], a more practical SIC receiver that initially ignores the energies received by all users and that estimates them featuring the subsequent techniques. Firstly, we consider an acquisition algorithm based on the known employed preambles to obtain the spreading code used by each user. Secondly, we estimate symbol energies of all users by preamble cross-correlations, after which users are ranked in non-increasing order of energy estimates. In this work, and for the sake of simplicity, we assume the receiver detects all users and focus this work on understanding the impact of estimation-based UO on SIC's performance. Hereinafter, we distinguish between the user-index $1 \leq k \leq K$ set in non-increasing order of received energies as (2), and the user-order $1 \leq k^{\prime} \leq K$ set according to energy estimates (3):

$$
\begin{aligned}
& E_{\mathrm{r}}[1] \geq E_{\mathrm{r}}[2] \geq \cdots \geq E_{\mathrm{r}}[k] \geq \cdots \geq E_{\mathrm{r}}[K], \\
& \hat{E}_{\mathrm{r}}[1] \geq \hat{E}_{\mathrm{r}}[2] \geq \cdots \geq \hat{E}_{\mathrm{r}}\left[k^{\prime}\right] \geq \cdots \geq \hat{E}_{\mathrm{r}}[K] .
\end{aligned}
$$

For user-packet demodulation, $K$-stage SIC is performed over $y(\mathrm{t})$ (1). At stage $k^{\prime}$ out of $K$, the spreading waveform $c_{k^{\prime}, m}\left(\mathrm{t}-\tau\left[k^{\prime}\right]\right)$ is employed as symbol matched filter to obtain the noisy symbol sequence associated with the $k^{\prime}$-th ordered user. Next, channel decoding operates, after which cyclic redundancy check, encapsulated next to information data, enables packet error detection. When it checks out, the decoded packet is regenerated and canceled from the received waveform. Otherwise, no cancellation is performed.

Our design focuses on the autonomous operation of network devices. We take advantage of slowly time-varying channels together with channel reciprocity to allow users $1 \leq k \leq K$ to accurately estimate their channel gains $h[1 \leq k \leq K]$ from a downlink pilot signal before transmission. This fact enables power control at transmitters, that may invert their channel gains so as to arrive at SIC with a specific energy distribution designed in this work. This simple scheme has an attractive practicality in satellite environments under SIC operation since the channel imbalance is usually in the same order as that needed by SIC to properly operate, and thus, channel inversion can be done with finite energy expenditure [11].

\section{A. System Model for Dynamically Ordered SIC}

We take up, for single-user packet decoding and cancellation, the system model adopted in [11], which generalizes the modeling of such operations for the short-packet communication and in which MA interference can be assumed
Gaussian after symbol despreading [3]. Hence, the packet error rate (PER) curve versus signal-to-interference-plus-noise ratio (SINR) after symbol despreading $\Gamma$ of the employed encoding system: PER $[\Gamma]$, is used to characterize the decoding stage, considering therein that every time a user is canceled an (average) fraction $\varepsilon(\Gamma)$ of its energy remains still uncanceled.

The authors in [14] suggest, as a shortcut to evaluating the long-term average system performance when handling a high number of users, the computation of statistical averages based on the probability $p_{k, k^{\prime}}$ that user $k$ is ordered the $k^{\prime}$-th, rather than the Monte Carlo method. $p_{k, k^{\prime}}$ denotes the user-order probability. Therein, the authors propose an average system model with the bivariate SINR and noise terms $\bar{\Gamma}\left[k, k^{\prime}\right]$ and $\bar{N}_{t}\left[k, k^{\prime}\right]$, standing for the corresponding utilities associated with user $k$ when ordered the $k^{\prime}$-th, which verify,

$$
\bar{\Gamma}\left[k, k^{\prime}\right]=E_{\mathrm{r}}[k] / \bar{N}_{t}\left[k, k^{\prime}\right] .
$$

For the computation of the average noise term $\bar{N}_{t}\left[k, k^{\prime}\right]$, we introduce the symbol decorrelation between non-orthogonal signatures $\frac{\theta}{N}$ [11], and we adopt the model for user $k$ when ordered at $k^{\prime}$ [14] in which, at previous stages $i<k^{\prime}$ the contribution of other users $u \neq k$ weighted by their user-order probabilities have been canceled. Then, we have

$$
\bar{\Gamma}\left[k, k^{\prime}\right]=\frac{E_{\mathrm{r}}[k]}{N_{0}+\frac{\theta}{N} \sum_{i<k^{\prime} u \neq k} \sum_{u \neq k} r[u, i] E_{\mathrm{r}}[u] p_{u, i}+\frac{\theta}{N} \sum_{i>k^{\prime} u \neq k} \sum_{\mathrm{r}}[u] p_{u, i}}
$$

with $r \triangleq 1-(1-\varepsilon(\Gamma)) \operatorname{PSR}[\Gamma]$ the average fraction of energy that remains after processing one user [11], [14], with $\operatorname{PSR}[\Gamma] \triangleq 1-\operatorname{PER}[\Gamma]$ the packet success rate function.

The user-aggregate $\mathrm{SE}$ is given then by the weighted sum

$$
\mathrm{SE} \triangleq \frac{R}{N} \sum_{k=1}^{K} \sum_{k^{\prime}=1}^{K} \operatorname{PSR}\left[\bar{\Gamma}\left[k, k^{\prime}\right]\right] p_{k, k^{\prime}}
$$

\section{ASYMPTOTIC SIC PERFORMANCE}

We analyze the user-limit case so as to approximate the behavior of a massively populated system by one with asymptotically many users. Then, $K \rightarrow \infty$ users transmit packets under very large spreading gains $N \rightarrow \infty$. This simple fact allows for a deterministic analysis as a function of the traffic load $\alpha=K / N$ [10], the user-index $t$ and the user-order $t^{\prime}$ [14]:

$$
t \triangleq \lim _{K \rightarrow \infty} K^{-1} k \quad, \quad t^{\prime} \triangleq \lim _{K \rightarrow \infty} K^{-1} k^{\prime} .
$$

Now, since users are condensed in the continuous interval $0 \leq t, t^{\prime} \leq 1$, asymptotic functions of the continuous argument $t$ such as $E_{\mathrm{r}}(t)$ can be defined in lieu of $E_{\mathrm{r}}[K t]$ (of a discrete argument $K t$ ), and for which $E_{\mathrm{r}}[K t] \rightarrow E_{\mathrm{r}}(t)$ as $K \rightarrow \infty$. Parameter $K$ is then obviated.

Moreover, the user-order probability $p_{k, k^{\prime}}$ now turned to the probability that user $t^{\prime}$ is ordered the $k^{\prime}$-th $\mathrm{d} p\left(t, t^{\prime}\right)$, is actually an infinitesimal quantity because each user can be ordered at $K \rightarrow \infty$ positions. Luckily, an explicit form for it is derived in [14] as a function of the probability distribution of energy estimates. For user $t$, energy estimation $\hat{E}_{\mathrm{r}}(t)=\beta^{-1} \mathcal{X}_{2}^{2}\left(\beta E_{\mathrm{r}}(t)\right)$ follows a scaled non-central chi squared distribution with 2 degrees of freedom and non-centrality parameter $\beta E_{\mathrm{r}}(t)$, with $\beta \triangleq 2 n_{o} /\left(N_{0}+\alpha \theta \bar{E}\right) . \bar{E} \triangleq \int_{0}^{1} E_{\mathrm{r}}(\tau) \mathrm{d} \tau$ is the average energy 
over the user population. Then, the respective density and tail distribution functions are given by

$$
\begin{aligned}
& f\left(E_{\mathrm{r}}, x\right)=\beta 2^{-1} \exp \left(-\beta 2^{-1}\left(x+E_{\mathrm{r}}\right)\right) I_{0}\left(\beta \sqrt{E_{\mathrm{r}} x}\right), \\
& \overline{\mathbb{F}}\left(E_{\mathrm{r}}, x\right)=\operatorname{Pr}\left[\mathcal{X}_{2}^{2}\left(\beta E_{\mathrm{r}}\right) \geq \beta x\right]=Q_{1}\left(\sqrt{\beta E_{\mathrm{r}}}, \sqrt{\beta x}\right) .
\end{aligned}
$$

where $Q_{1}(a, b)$ is the Marcum Q function of first order, and $I_{\rho}(x)$ the modified Bessel function of first kind and order $\rho$.

Then, we finally have [14]

$$
\frac{\mathrm{d} p\left(t, t^{\prime}\right)}{\mathrm{d} t^{\prime}}=\frac{\mathrm{d} \overline{\mathbb{F}}\left(E_{\mathrm{r}}(t), \mu^{-1}\left(t^{\prime}\right)\right)}{\mathrm{d} t^{\prime}}, \mu(x)=\int_{0}^{1} \overline{\mathbb{F}}\left(E_{\mathrm{r}}(t), x\right) \mathrm{d} t
$$

where, by expanding the left part of (9) we obtain $\mathrm{d} p\left(t, t^{\prime}\right)=$ $a\left(t, t^{\prime}\right) \mathrm{d} t^{\prime}$, with $a\left(t, t^{\prime}\right)$ the $K$-independent kernel

$$
a\left(t, t^{\prime}\right)=-f\left(E_{\mathrm{r}}(t), \mu^{-1}\left(t^{\prime}\right)\right) / \dot{\mu}\left(\mu^{-1}\left(t^{\prime}\right)\right) .
$$

\section{A. Asymptotic Utility Functions}

We use the previous equations to address some simplifications next. The SINR utility $\bar{\Gamma}\left[k, k^{\prime}\right](4)$ is now turned to a separable function of $t$ and $t^{\prime}$ as $\bar{\Gamma}\left(t, t^{\prime}\right)=E_{\mathrm{r}}(t) / \bar{N}_{t}\left(t^{\prime}\right)$. Then:

1) The noise plus interference term $\bar{N}_{t}\left(t^{\prime}\right)$ is the univariate function of the user-order $t^{\prime}$

$$
\begin{aligned}
\bar{N}_{t}\left(t^{\prime}\right) & =N_{0}+\alpha \theta \int_{0}^{t^{\prime}} \int_{0}^{1} r(u, \tau) E_{\mathrm{r}}(u) a(u, \tau) \mathrm{d} u \mathrm{~d} \tau \\
& +\alpha \theta \int_{t^{\prime}}^{1} \int_{0}^{1} E_{\mathrm{r}}(u) a(u, \tau) \mathrm{d} u \mathrm{~d} \tau .
\end{aligned}
$$

The first noise term is $\bar{N}_{t}(0)=N_{0}+\alpha \theta \bar{E}$. It is worth noting that the stated system model is an extension of that in [11] where single integrals have been substituted by double integrals that average the contributions of all users. We then obtain a new differential equation that models the evolution of the average noise term under a dynamically ordered SIC. Applying the gradient operator $\nabla_{t^{\prime}}(\cdot)$ and dividing at both sides by $\bar{N}_{t}\left(t^{\prime}\right)$ we get

$$
\frac{\dot{\bar{N}}_{t}\left(t^{\prime}\right)}{\bar{N}_{t}\left(t^{\prime}\right)}=-\alpha \int_{0}^{1} \Phi\left[\bar{\Gamma}\left(u, t^{\prime}\right)\right] a\left(u, t^{\prime}\right) \mathrm{d} u .
$$

$\Phi[\Gamma] \triangleq \theta(1-\varepsilon(\Gamma)) \Gamma \cdot \operatorname{PSR}[\Gamma]$ is analogous to [11].

2) Asymptotic $S E$ : The user-aggregate $S E$ is now given in the user-asymptotic case by the double integral

$$
\mathrm{SE}=\alpha R \int_{0}^{1} \int_{0}^{1} \operatorname{PSR}\left[\bar{\Gamma}\left(t, t^{\prime}\right)\right] a\left(t, t^{\prime}\right) \mathrm{d} t \mathrm{~d} t^{\prime} .
$$

\section{Optimization of the Dynamically ORdered SIC}

In this section, we address system optimization for determining the most favorable asymptotic energy function $E_{\mathrm{r}}(t)$ that maximizes the SE (13) under the average energy constraint

$$
\int_{0}^{1} E_{\mathrm{r}}(t) \mathrm{d} t=\bar{E}
$$

which can be contextualized in some satellite settings to constrain energy expenditure [11]. First of all, we manipulate previous equations to simplify the posterior optimization. We are interested in rewriting (12)-(13), which depend on $a\left(t, t^{\prime}\right)$ (10), in a way so as to suppress the dependence on the inverse function $\mu^{-1}\left(t^{\prime}\right)$. The subsequent steps are followed:
1) Noise plus interference term: We apply over (12) the change of variable $t^{\prime}=\mu(x)$ and define the noise term $\tilde{N}_{t}(x) \triangleq \bar{N}_{t}(\mu(x))$, for which (12) admits the following simplified version with the initial term $\tilde{N}_{t}(\infty)=\bar{N}_{t}(0)=N_{0}+\alpha \theta \bar{E}$ :

$$
\frac{\dot{\tilde{N}}_{t}(x)}{\tilde{N}_{t}(x)}=\alpha \int_{0}^{1} \Phi\left[\frac{E_{\mathrm{r}}(t)}{\tilde{N}_{t}(x)}\right] f\left(E_{\mathrm{r}}(t), x\right) \mathrm{d} t,
$$

2) Asymptotic SE: The same change of variable is applied over (13) under the differential $\mathrm{d} x=\left[\dot{\mu}\left(\mu^{-1}\left(t^{\prime}\right)\right)\right]^{-1} \mathrm{~d} t^{\prime}$, so that $a\left(t, t^{\prime}\right) \mathrm{d} t^{\prime}$ is substituted by $-f\left(E_{\mathrm{r}}(t), x\right) \mathrm{d} x$ with the integral limits $\mu^{-1}(0)=\infty$ and $\mu^{-1}(1)=0$, as:

$$
\mathrm{SE}=\alpha R \int_{0}^{\infty} \int_{0}^{1} \operatorname{PSR}\left[\frac{E_{\mathrm{r}}(t)}{\tilde{N}_{t}(x)}\right] f\left(E_{\mathrm{r}}(t), x\right) \mathrm{d} t \mathrm{~d} x .
$$

\section{A. Variational Calculus Problem}

We remind that we want to maximize SE (16) under the average energy constraint (14) provided that the sought userenergy distribution $E_{\mathrm{r}}(t)$ generates such a $\tilde{N}_{t}(x)$ satisfying the dynamic equation (15). We have the optimization problem

$$
\max _{E_{\mathrm{r}}(t), \tilde{N}_{t}(x)} \mathrm{SE} \quad \text { s.t. } \quad \text { eqs. }(14)-(15) .
$$

For better readability, since all the expressions only depend on $E_{\mathrm{r}}(t)$ and $\tilde{N}_{t}(x)$, hereafter, we consider them denoted $E_{\mathrm{r}}$ and $\tilde{N}_{t}$, and the following definitions:

$$
\begin{aligned}
& A\left[E_{\mathrm{r}}, \tilde{N}_{t}, x\right] \triangleq \operatorname{PSR}\left[E_{\mathrm{r}} / \tilde{N}_{t}\right] \cdot f\left(E_{\mathrm{r}}, x\right), \\
& B\left[E_{\mathrm{r}}, \tilde{N}_{t}, x\right] \triangleq \Phi\left[E_{\mathrm{r}} / \tilde{N}_{t}\right] \cdot f\left(E_{\mathrm{r}}, x\right) .
\end{aligned}
$$

With regard to $E_{\mathrm{r}}$, we adopt discontinuous or broken extremal [15, Ch. 3.15] solutions to our problem, more concretely, piecewise continuously differentiable candidates comprising a finite number of pieces $p$. Piece $k$ is indicated as $E_{\mathrm{r}}^{k}(t)$, or simply $E_{\mathrm{r}}^{k}$. Regarding the term $\tilde{N}_{t}$, we consider continuously differentiable solutions in $x \geq 0$. Then, we resort to variational calculus tools to derive the stationary points of (17).

Therefore, we formulate the Lagrangian for a fixed partition of the variable $0 \leq t \leq 1, t_{0}, \ldots, t_{p}$ with $t_{0}=0$ and $t_{p}=1$. We impose the constraint (14) using the scalar $\lambda$, and the equation (15) for each $x \geq 0$ using $\beta(x)$. Then,

$$
\begin{aligned}
\mathcal{L} & \triangleq \int_{0}^{\infty} \sum_{k=1}^{p} \int_{t_{k-1}}^{t_{k}} A\left[E_{\mathrm{r}}^{k}, \tilde{N}_{t}, x\right] \mathrm{d} t \mathrm{~d} x-\lambda\left(\sum_{k=1}^{p} \int_{t_{k-1}}^{t_{k}} E_{\mathrm{r}}^{k} \mathrm{~d} t-\bar{E}\right) \\
& -\int_{0}^{\infty} \beta(x)\left(\frac{\tilde{\tilde{N}}_{t}}{\tilde{N}_{t}}-\alpha \sum_{k=1}^{p} \int_{t_{k-1}}^{t_{k}} B\left[E_{\mathrm{r}}^{k}, \tilde{N}_{t}, x\right] \mathrm{d} t\right) \mathrm{d} x .
\end{aligned}
$$

We follow the rationales in [15] to solve it in each of the adopted function spaces. The extensive formal proof is omitted due to lack of space, and only its main steps are shown. Firstly, the Euler-Lagrange operator $\left(\partial_{E_{\mathrm{r}}}-\nabla_{t} \partial_{\dot{E}_{\mathrm{r}}}\right)(\cdot)$ is applied over the integrand associated with variable $t$ to obtain the equation that the stationary $E_{\mathrm{r}}^{k}$ must satisfy:

$$
\int_{0}^{\infty}\left[\partial_{E_{\mathrm{r}}} A+\alpha \beta(x) \partial_{E_{\mathrm{r}}} B\right] \mathrm{d} x=\lambda \quad \text { in } \quad t_{k-1} \leq t<t_{k}
$$

The explicit form for partial derivatives is omitted. 


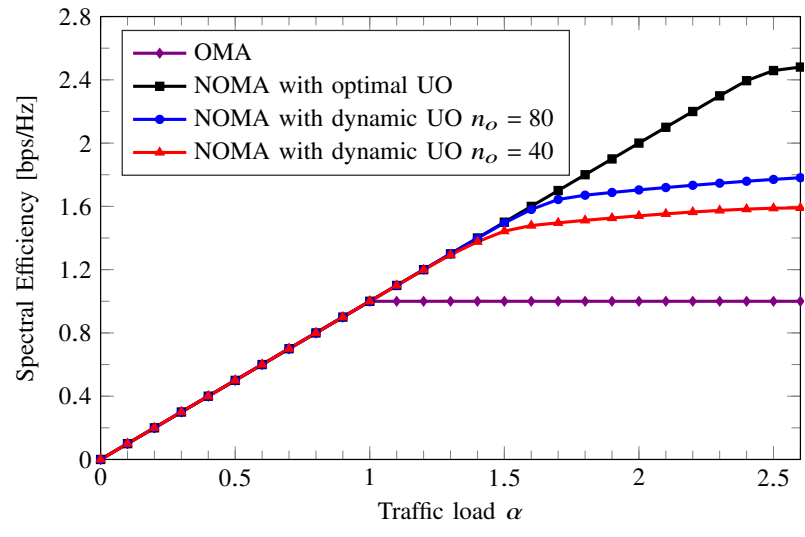

Fig. 1. Spectral efficiency versus traffic load $\alpha$.

Secondly, $\left(\partial_{\tilde{N}_{t}}-\nabla_{x} \partial_{\dot{N}_{t}}\right)(\cdot)$ is applied over the integrand w.r.t. $x$ so as to obtain the condition for the stationary $\tilde{N}_{t}$ :

$$
\sum_{k=1}^{p} \int_{t_{k-1}}^{t_{k}}\left[A_{\tilde{N}_{t}}+\alpha \beta(x) B_{\tilde{N}_{t}}\right] \mathrm{d} t=\dot{\beta}(x) / \tilde{N}_{t} \quad \text { in } \quad x \geq 0
$$

with the initial value $\dot{\beta}(0)=0$. Now, we discuss the obtained results. Specifically, we focus on the stationary $E_{\mathrm{r}}^{k}$ in (21), which integrates the bivariate function $\partial_{E_{\mathrm{r}}} A+\alpha \beta(x) \partial_{E_{\mathrm{r}}} B$ under $x$. This gives, by defining the non-linear function $G$, the result of the left part of (21), and by recovering the argument $t$

$$
G\left(E_{\mathrm{r}}^{k}(t)\right)=\lambda \text { in } t_{k-1} \leq t<t_{k} .
$$

Since the former needs to be satisfied in $t_{k-1} \leq t<t_{k}$, the only solution is to have intervals with constant ( $t$-independent) levels $E_{\mathrm{r}}^{k}(t)=E_{\mathrm{r}}^{k}$. Then, the stationary $E_{\mathrm{r}}(t)$ obey piecewise constant structures regardless of the partition of $t$.

\section{B. Vector Calculus Problem}

Due to the complexity of determining the globally optimal solution, we propose a complexity-affordable search to determine a competitive locally-optimal solution.

We particularize (17) with the stationary structure comprising $p$ constant pieces. Therefore, we address in (25a)-(25e) two optimizations. Firstly, for a temporary value of $p$, we solve the inner vector optimization under

$$
\boldsymbol{E}_{\mathbf{r}} \triangleq\left[E_{\mathrm{r}}^{1}, E_{\mathrm{r}}^{2}, \ldots, E_{\mathrm{r}}^{p}\right]^{T}, \Delta \boldsymbol{\Delta} \triangleq\left[\Delta t_{1}, \Delta t_{2}, \ldots, \Delta t_{p}\right]^{T} .
$$

Secondly, the remaining parameter to determine is the number of pieces $p$, and that, as it can only take discrete values, we address by enumerative search in the outer problem. We have:

$$
\begin{aligned}
\max _{p \geq 1} \max _{\boldsymbol{E}_{\mathbf{r}}, \Delta \boldsymbol{t}} & \int_{0}^{\infty} \boldsymbol{\Delta} \boldsymbol{t}^{T} A\left[\boldsymbol{E}_{\mathbf{r}}, \tilde{N}_{t}(x), x\right] \mathrm{d} x \\
\text { s.t. } & \bar{E}=\boldsymbol{\Delta} \boldsymbol{t}^{T} \boldsymbol{E}_{\mathbf{r}} \quad, \quad 1=\boldsymbol{\Delta} \boldsymbol{t}^{T} \mathbf{1}_{p} \\
\text { s.t. } & \tilde{N}_{t}(x) \\
\bar{N}_{t}(0) & \exp \left(-\alpha \int_{x}^{\infty} \boldsymbol{\Delta} \boldsymbol{t}^{T} B\left[\boldsymbol{E}_{\mathbf{r}}, \tilde{N}_{t}(u), u\right] \mathrm{d} u\right) \\
\text { s.t. } & \boldsymbol{E}_{\mathbf{r}} \geq \mathbf{0}_{p} \quad, \quad \boldsymbol{\Delta} \boldsymbol{t}>\mathbf{0}_{p} \\
\text { s.t. } & E_{\mathrm{r}}^{i} \geq E_{\mathrm{r}}^{i+1}, \quad 1 \leq i \leq p-1
\end{aligned}
$$

In the previous problem, we have turned the differential equation (15) to the integral equation (25c), and we have incorporated the constraint (25e) to consider ordered solutions. More details about its numerical resolution are addressed next.

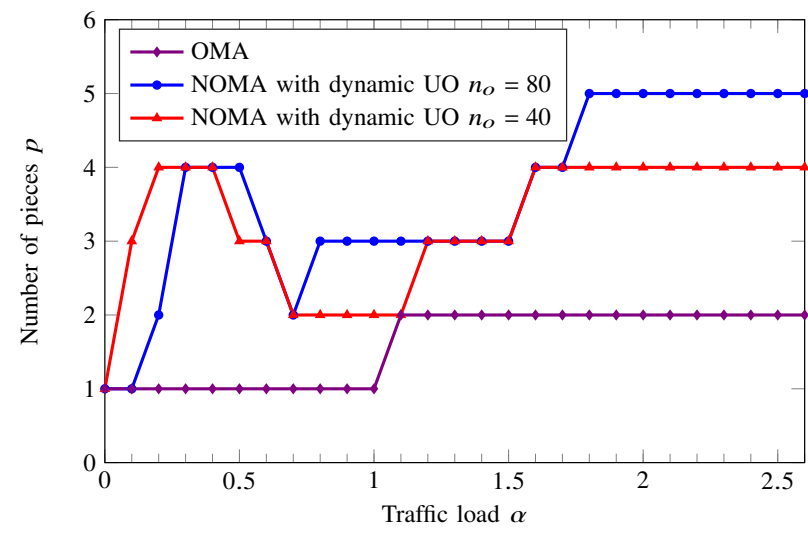

Fig. 2. Effective number of pieces $p$ versus traffic load $\alpha$.

\section{Numerical Implementation and Computational Benefits}

Even if we reduce the searching from a function space to a parameter space, (25a)-(25b) is also complex.

The inner problem in (25a)-(25e) is non-linear and nonconvex since it depends on the specific forms of PSR $[\Gamma]$ and $\Phi[\Gamma]$, which present several intervals of concavity. We follow an easy strategy with a high performance/complexity ratio: we solve it using sequential quadratic programming, where local-optimality is ensured by checking the Hessian, we repeat the algorithm $N_{\text {init }}$ times with random initialization of $\boldsymbol{E}_{\mathbf{r}}$ and $\Delta t$, and keep the solution that achieves higher SE. Moreover, to simplify its numerical evaluation, integrals are turned to Riemann sums in $x \in\left[0, x_{\max }\right]$ with $r$ intervals: $x_{1}, \ldots, x_{r}$. The noise terms $\tilde{N}_{t}\left(x_{1 \leq i \leq r}\right)$ are computed recursively as follows: (i) $\tilde{N}_{t}\left(x_{r}\right)=\bar{N}_{t}(0)$ for $x_{r}=x_{\max }$; and (ii) $\tilde{N}_{t}\left(x_{i-1}\right)=\tilde{N}_{t}\left(x_{i}\right)$. $\exp \left(-\frac{\alpha}{r} \boldsymbol{\Delta} \boldsymbol{t}^{T} B\left[\boldsymbol{E}_{\mathbf{r}}, \tilde{N}_{t}\left(x_{i}\right), x_{i}\right]\right)$ for $i=2, \ldots, r$.

Regarding the outer optimization, we incorporate a halting policy to stop the enumerative search in $p$ when, for increasing $p$, we experiment insignificant changes to the locally-optimal solution. We stop the search in $p$ when we obtain for two consecutive $p$-values singular solutions having $E_{\mathrm{r}}^{i} \cong E_{\mathrm{r}}^{j}$ for some $i, j$, or some $\Delta t_{k} \approx 0$.

\section{Simulation Results}

We have considered a short-packet communication setting where users use payload $n_{e}=360$ and preamble lengths (in symbols) 40 and 80 , which constitute the $10 \%$ and $19 \%$ of the total packet duration. Coding and modulation are jointly characterized by a PER vs. SINR curve PER $[\Gamma]$ extracted from the maximal channel coding rate at blocklength $n_{e}$ and the encoding rate $R=1 \mathrm{bps} / \mathrm{Hz}$. For validation we consider, as in [11]-[13], a SINR-independent uncanceled energy fraction $\varepsilon(\Gamma)=0.01$ that models systematic inaccuracies in waveform reconstruction. The decorrelation factor $\theta$ is set to 1 , and the average energy constraint $\bar{E} / N_{0}=10 \mathrm{~dB}$.

The first part of this section comprises a theoretical study of the distributions computed under rationales in Section IV-C with $r=1000$ points, $x_{\max }=100$ and $N_{\text {init }}=10$. Fig. 1 depicts the SE for perfectly [11] (square) and dynamically (circle, triangle) ordered SIC. The maximum SE degradation for preambles of 40 and 80 symbols is about $35 \%$ and $28 \%$, 


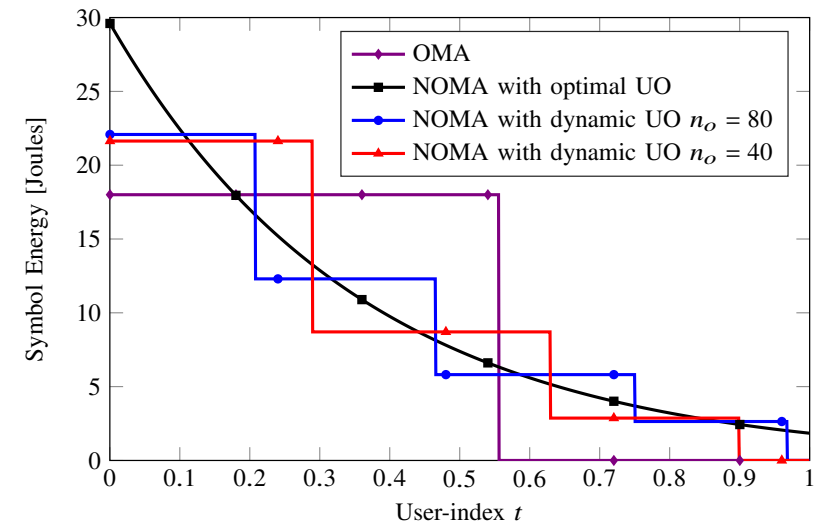

Fig. 3. User-energy distribution $E_{\mathrm{r}}(t)$ at the (high) activity load $\alpha=1.80$.

respectively. SE strongly depends on the variance of userenergy estimations at high $\alpha$, whereas imperfect estimations cause negligible detriment on SE at low $\alpha$. NOMA shows significant SE improvement relative to OMA.

We draw in Fig. 2 the effective (after correcting singularities) number of pieces that comprise the locally optimal distributions. The uniform distribution $E_{\mathrm{r}}(t)=\bar{E}(p=1)$ is only optimal at $\alpha=0$, whereas more pieces appear at increasing $\alpha$. A complex interaction exists between: (i) increasing $p$ to approach the distribution with optimal UO and thus, improve SIC's performance; and (ii) reducing $p$ to diminish the impact of energy estimations on the UO establishment.

The obtained user-energy distributions are depicted in Fig. 3 for a single traffic load. The optimum $E_{\mathrm{r}}(t)$ under optimal UO is almost exponential (square) [11]. When the UO is set as a function of energy estimates in a large network, it is better, instead of allocating exponentially decaying energies [10], [11], to conform groups of users under equal energy. An intuitive rationale for that piecewise constant structure is that the estimation of the strongest user (a single user) is equivalent to the estimation of any user from the same group. For the cases studied in Fig. 3, we found $p=4$ pieces for $n_{o}=40$ (triangle), with the last level set to zero so as to avoid the transmission of $10 \%$ of users. For $n_{o}=80$ symbols (circle), we found $p=5$. In the OMA case, energy estimations do not affect SE, which is optimized under $E_{\mathrm{r}}(t)=\bar{E} / t_{*}$ in $0 \leq t<t_{*}$ and $E_{\mathrm{r}}(t)=0$ in $t_{*} \leq t \leq 1$ with $t_{*}=\min \{1,1 / \alpha\}$.

The second part of this section assesses, empirically, the behavior of SIC for the distribution depicted in Fig. 3 using red diamonds. We average $10^{4}$ runs of the SIC implementation in [14, Section II-C]: (i) estimate $K$ symbol energies from $n_{o^{-}}$ symbol preambles; (ii) order users as (3); and (iii) perform SIC. The user-asymptotic behavior accurately predicts the empirical PERs for $K \geq 115$ users.

\section{CONCLUSION}

We have analyzed a large-user network transmitting packets towards a practical gateway performing SIC. We have considered the same codebook for all users together with random spreading signatures, and that the resulting multiple access interference at the output of the matched filter follows a Gaussian distribution. For user-packet demodulation, we have

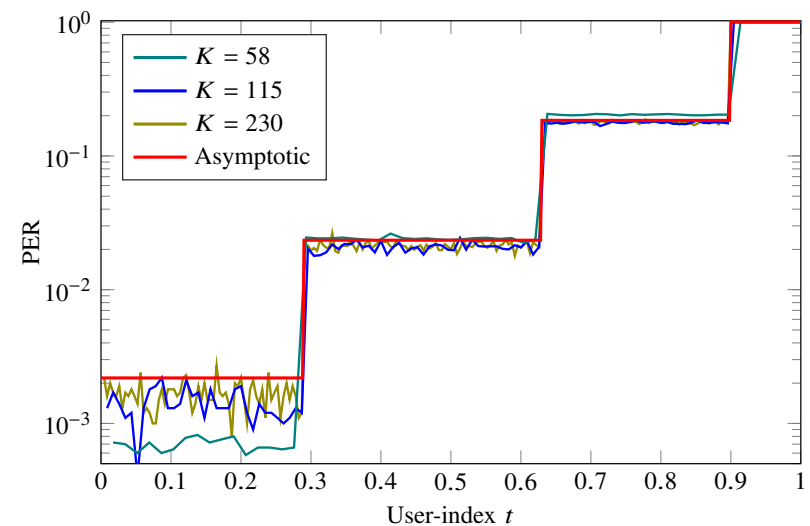

Fig. 4. Individual performances in terms of the PER each user experiments.

adopted a SIC gateway that estimates energies of all users at the initial stage by preamble cross-correlations, and proceeds in non-increasing order of the former estimates. We have analyzed the user-asymptotic regime and solved a variational problem that concludes that the user-energy distribution at reception that maximizes the SIC's performance (spectral efficiency) is a piecewise constant function defined from a finite set of energy levels. Yet, an analytic expression or bound to the globally optimal number of pieces remains to be found.

\section{REFERENCES}

[1] Z. Ding, R. Schober, and H. V. Poor, "Unveiling the importance of SIC in NOMA systems: Part I - state of the art and recent findings," IEEE Commun. Lett., vol. 24, no. 11, pp. 2373-2377, 2020.

[2] G. Song, X. Wang, and J. Cheng, "A low-complexity multiuser coding scheme with near-capacity performance," IEEE Trans. Veh. Technol., vol. 66, no. 8, pp. 6775-6786, Aug. 2017.

[3] S. Verdú, Multiuser detection. Cambridge university press, 1998.

[4] A. Suarez, M. Debbah, L. Cottatellucci, and E. Altman, "Optimal decoding order under target rate constraints," in 2007 IEEE 8th Workshop on Signal Processing Advances in Wireless Commun., Jun. 2007, pp. 1-5.

[5] Y. Polyanskiy, "A perspective on massive random-access," in 2017 IEEE Int. Symp. Inf. Theory (ISIT), Jun. 2017, pp. 2523-2527.

[6] A. U.Toboso, S. Loyka, and F. Gagnon, "Optimal detection ordering for coded V-BLAST," IEEE Trans. Commun., vol. 62, no. 1, pp. 100-111, Jan. 2014

[7] B.Xia, J.Wang, K. Xiao, Y.Gao, Y. Yao, and S. Ma, "Outage performance analysis for the advanced SIC receiver in wireless NOMA systems," IEEE Trans. Veh. Technol., vol. 67, no. 7, pp. 6711-6715, Jul. 2018.

[8] Y. Gao, B. Xia, Y. Liu, Y. Yao, K. Xiao, and G. Lu, "Analysis of the dynamic ordered decoding for uplink NOMA systems with imperfect CSI," IEEE Trans. Veh. Technol., vol. 67, no. 7, pp. 6647-6651, Jul. 2018.

[9] R. De Gaudenzi, O. DR Herrero, and G. Gallinaro, "Enhanced spread ALOHA physical layer design and performance," Int. Journal on Satellite Communications and Networking, vol. 32, no. 6, pp. 457-473, 2014.

[10] D. V. Djonin and V. K. Bhargava, "Asymptotic analysis of the conventional decision feedback receiver in fading channels," IEEE Trans. Wireless Commun., vol. 2, no. 5, pp. 1066-1078, Sep. 2003.

[11] J. Sala-Álvarez, F. Rey, J. Villares, and F. Molina, "Minimum PER user-energy profile for massive SIC receivers under an average energy constraint," in 2017 IEEE 18th Int. Workshop on Signal Processing Advances in Wireless Commun., Jul. 2017, pp. 1-6.

[12] J. G. Andrews and T. H. Meng, "Optimum power control for successive interference cancellation with imperfect channel estimation," IEEE Trans. Wireless Commun., vol. 2, no. 2, pp. 375-383, Mar. 2003.

[13] F. Collard and R. De Gaudenzi, "On the optimum packet power distribution for spread ALOHA packet detectors with iterative successive interference cancelation," IEEE Trans. Wireless Commun., vol. 13, no. 12, pp. 6783-6794, Dec. 2014.

[14] F. Molina and J. Sala-Álvarez, "Asymptotic performance analysis of successive interference cancellation with dynamic user-decoding order," IEEE Commun. Lett., vol. 24, no. 12, pp. 2931-2935, 2020.

[15] I. M. Gelfand et al., Calculus of variations. Courier Corporation, 2000. 